\title{
Modules that have a Rad-supplement in every cofinite extension
}

\author{
Burcu Nişancı Türkmen
}




\title{
MODULES THAT HAVE A RAD-SUPPLEMENT IN EVERY COFINITE EXTENSION
}

\author{
BURCU NIŞANCI TÜRKMEN
}

Received 30 January, 2013

\begin{abstract}
In this paper, we study modules with the properties (CRE) and (CREE), which are adapted Zöschinger's modules with the properties $(E)$ and $(E E)$. It is shown that: (1) a module $M$ has the propery (CREE) if and only if every submodule of $M$ has the propery (CRE); (2) a ring $R$ is $R a d$-supplemented if and only if every left $R$-module has the propery (CRE); (3) over a commutative Von Neumann regular ring a module $M$ has the propery (CRE) if and only if $M$ is cofinitely injective.
\end{abstract}

2010 Mathematics Subject Classification: 16D10; 16 N80

Keywords: Rad-supplement, cofinite extension, Von Neumann regular ring, semiperfect ring

\section{INTRODUCTION}

In this note, $R$ is an associative ring with identity and all modules are unital left $R$ modules. Let $N$ be an $R$-module. The notation $M \subseteq N$ means that $M$ is a submodule of $N$. Here, $N$ is called an extension of $M$. A module $M$ is called injective if $M$ is a direct summand of every extension $N$, i.e. $N=M+K$ and $N \cap K=0$ for some submodule $K \subseteq N$. If every $R$-module is injective, then $R$ is semisimple. The converse is true.

A submodule $S$ of a module $M$ is said to be small if $S+L \neq M$ for every proper submodule $L$ of $M$. For every module $M$, the trivial submodule 0 is a small submodule of $M$. As a generalization of direct summands, we say that a submodule $V$ of a module $M$ is supplement of a submodule $U$ of $M$ if $M=U+V$ and $U \cap V \ll V$ as in [11]. Zöschinger generalized injective modules to modules with the property (E). He said that a module $M$ has the property (E) if $M$ has a supplement in every extension. He also said that a module $M$ has the property (EE) if $M$ has ample supplements in every extension, i.e. for $M \subseteq N$, if $N=M+K, K$ contains a supplement of $M$ in $N$ ([13]). Every left $R$-module has the property $(E)$ if and only if $R$ is left perfect [4].

In [1], a submodule $M$ of a module $N$ is called cofinite if the factor module $\frac{N}{M}$ is finitely generated. It is easy to see that every submodule of a finitely generated module is cofinite. Adapting Zöschinger's module with the properties (E) and (EE), 
Çalışıcı and Türkmen say that a module $M$ has the property (CE) ((CEE)) if $M$ has a supplement (ample supplements) in every cofinite extension. Here $N$ is called cofinite extension of $M$ if $M$ is a cofinite submodule of $N$. They give a characterization of semiperfect rings via these modules.

For a module $M, \operatorname{Rad}(M)$ will indicate the Jacobson radical of $M$. A submodule $V$ of a module $M$ is called Rad-supplement of a submodule $U$ in $M$ if $M=U+V$ and $U \cap V \subseteq \operatorname{Rad}(V)$ [4, Theorem 10.14]. Since the Jacobson radical of a module is the sum of all small submodules, every supplement is $R a d$-supplement. A submodule $U$ of $M$ has ample $R a d$-supplements in $M$ if every submodule $V$ of $M$ with $M=U+V$ contains a $R a d$-supplement in $M$. Now we have the following implications on submodules:

$$
\text { direct summand } \Longrightarrow \text { supplement } \Longrightarrow \text { Rad-supplement }
$$

In this paper, we investigate the properties (CRE) and (CREE) for modules as a generalization of the properties (CE) and (CEE), respectively. We show that a module has the property (CREE) if and only if every submodule has the property (CRE). We prove that every direct summand of a module $M$ with the property (CRE) has this property. Using the property (CRE), we give a characterization of Radsupplemented rings, which are a generalization of semiperfect rings.

\section{Modules With the PROperties (CRE) AND (CREE)}

In this section, we define the concept of modules with the properties (CRE) and (CREE). We give various properties of these modules. We prove that every left $R$ module has the property (CRE) if and only if the ring $R$ is $R a d$-supplemented.

Definition 1. Let $M$ be an $R$-module. We say that $M$ has the property (CRE) if $M$ has a $R a d$-supplement in every cofinite extension, and $M$ has the property (CREE) if $M$ has ample $R a d$-supplements in every cofinite extension

It is easy to see that every module with property (CE) has property (CRE). It is natural to pose the question whether there exists similar result for the properties (CEE) and (CREE). Before the end of this section, we shall give an example of a module which has the property (CRE) but not (CE).

Proposition 1. Every radical module has the property (CRE).

Proof. Let $M$ be a module and $N$ be any cofinite extension of $M$. Since $\operatorname{Rad}(M)=$ $M$, then $N=M+N$ and $M \cap N=M=\operatorname{Rad}(M) \subseteq \operatorname{Rad}(N)$. So $N$ is a $\operatorname{Rad}$ supplement of $M$ in $N$.

By $P(M)$, we denote the sum of all radical submodules of the module $M$, that is, $P(M)=\sum\{U \subseteq M \mid \operatorname{Rad}(U)=U\}$. A module $M$ is reduced if $P(M)=0$.

Since $P(M)$ is a radical submodule of $M$, we have the next result which is a direct consequence of Proposition 1. 
Corollary 1. For a module $M, P(M)$ has the property (CRE).

Now we show that the property (CRE) is preserved by direct summands in the following proposition:

Proposition 2. Every direct summand of a module with the property (CRE) has the property (CRE).

Proof. Let $N$ be a direct summand of $M$. Then there exists a submodule $K$ of $M$ such that $M=N \oplus K$. Let $L$ be a cofinite extension of $N, T$ be the external direct sum $L \oplus K$ and $\gamma: M \longrightarrow T$ be the canonical embedding. Then $M \cong \gamma(M)$ has the property (CRE). We have

$$
\frac{L}{N} \cong \frac{L \oplus K}{\gamma(M)}
$$

is finitely generated. Since $\gamma(M)$ has the property (CRE), then there exists a submodule $U$ of $T$ such that $T=\gamma(M)+U$ and $\gamma(M) \cap U \subseteq \operatorname{Rad}(U)$. Consider the projection $\pi: T \longrightarrow L$. By this way, we have $N+\pi(U)=L$. Also $\operatorname{ker}(\pi) \subseteq \gamma(M)$, $\pi(\gamma(M) \cap U) \subseteq \pi(\gamma(M)) \cap \pi(U)=N \cap \pi(U) \subseteq \operatorname{Rad}(\pi(U))$. Therefore $\pi(U)$ is a $R a d$-supplement of $N$ in $L$.

In [3], Çalışıcı and Türkmen defined cofinitely injective modules, that is, a module $M$ is called cofinitely injective if $M$ is a direct summand of every cofinite extension.

Recall that a ring $R$ is called Von Neumann regular if every element $a \in R$ can be written in the form axa, for some $x \in R$.

Proposition 3. Let $R$ be a commutative Von Neumann regular ring and $M$ be an $R$-module. Then the following statements are equivalent:

(1) $M$ has the property (CRE).

(2) $M$ is cofinitely injective.

(3) $M$ has the property (CE).

Proof. (1) $\Longrightarrow(2)$ Suppose that $M$ is a module with the property (CRE). Let $N$ be any cofinite extension of $M$. So, there exists a $\operatorname{Rad}$-supplement $V$ of $M$ in $N$. Since $R$ is a commutative Von Neumann regular ring, $\operatorname{Rad}(V)=0$ by $[5,3.73$ and 3.75]. So $N=M \oplus V$.

(2) $\Longrightarrow(3)$ and (3) $\Longrightarrow(1)$ are clear.

Zöschinger proved in [13] that a module has the property (EE) if and only if every submodule has the property (E). We give an analogous characterization of our modules.

Theorem 1. A module $M$ has the property (CREE) if and only if every submodule of $M$ has the property (CRE). 
Proof. ( $\Longrightarrow$ ) Let $M$ be a module with the property (CREE) and $T$ be any submodule of $M$. For a cofinite extension $N$ of $T$, let $F=\frac{M \oplus N}{H}$, where the submodule $H$ is the set of all elements $(a,-a)$ of $F$ with $a \in T$ and let $\alpha: M \longrightarrow F$ via $\alpha(m)=(m, 0)+H, \beta: N \longrightarrow F$ via $\beta(n)=(0, n)+H$ for all $m \in M, n \in N$. It is clear that $\alpha$ and $\beta$ are monomorphisms. Thus we have the following pushout:

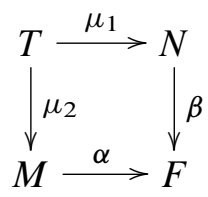

where $\mu_{1}$ and $\mu_{2}$ are inclusion mappings. It is easy to prove that $F=\operatorname{Im}(\alpha)+$ $\operatorname{Im}(\beta)$. Now we define $\gamma: F \longrightarrow \frac{N}{T}$ by $\gamma((m, n)+H)=n+T$ for all $(m, n)+H \in$ $F$. Then $\gamma$ is an epimorphism. Note that $\operatorname{ker}(\gamma)=\operatorname{Im}(\alpha)$ and so $\frac{N}{T} \cong \frac{F}{\operatorname{Im}(\alpha)}$ is finitely generated. Since $\alpha$ is a monomorphism, we have $M \cong \operatorname{Im}(\alpha)$. By the assumption, $\operatorname{Im}(\alpha)$ has the property (CREE). Then it follows immediately that $\operatorname{Im}(\alpha)$ has a Rad-supplement $V$ in $F$ with $V \subseteq \operatorname{Im}(\alpha)$, i.e., $F=\operatorname{Im}(\alpha)+V$ and $\operatorname{Im}(\alpha) \cap V \subseteq$ $\operatorname{Rad}(V)$. Then $N=T+\beta^{-1}(V)$ and $T \cap \beta^{-1}(V) \subseteq \operatorname{Rad}\left(\beta^{-1}(V)\right)$. Hence $\beta^{-1}(V)$ is a $R a d$-supplement of $T$ in $N$.

( $\Longleftarrow$ ) Suppose that every submodule of $M$ has the property (CRE). For a cofinite extension $N$ of $M$, let $N=M+K$ for some submodule $K$ of $N$. Then $\frac{N}{M} \cong \frac{K}{M \cap K}$ is finitely generated and so $M \cap K$ is a cofinite submodule of $K$. By the hypothesis, there exists a submodule $V$ of $K$ such that $K=(M \cap K)+V$ and $M \cap V \subseteq \operatorname{Rad}(V)$. Note that $N=M+V$. It follows that $V$ is a $R a d$-supplement of $M$ in $N$. So $M$ has the property (CREE).

Recall from [2] that a module $M$ is (cofinitely) Rad-supplemented if every (cofinite) submodule has a $R a d$-supplement in $M$. It is clear that every finitely generated cofinitely Rad-supplemented module is Rad-supplemented.

Corollary 2. Every submodule of a module with the property (CREE) has the property (CRE). Moreover, a module with the property (CREE) is cofinitely Radsupplemented.

Proposition 4. Let $M$ be a module and $A$ be a submodule of $B$ such that $\frac{B}{A}$ is noetherian. If $A$ and $\frac{B}{A}$ have the property (CRE), then so is $B$.

Proof. Let $N$ be any cofinite extension of $B$. By the hypothesis, there is a Radsupplement $\frac{V}{A}$ of $\frac{B}{A}$ in $\frac{N}{A}$. Note that $\frac{N}{B} \cong \frac{\frac{V}{A}}{\frac{B \cap V}{A}}$. Since $\frac{B}{A}$ is noetherian, then $\frac{V}{A}$ is finitely generated. Therefore $V$ is a cofinite extension of $A$. Since $A$ has the property (CRE), then $A$ has a $R a d$-supplement in $V$, say $K$. We claim that $K$ is a $R a d$-supplement of $B$ in $N$. We have epimorphisms $f: K \longrightarrow \frac{V}{A}$ and $g: \frac{V}{A} \longrightarrow \frac{N}{B}$ such that $\operatorname{ker}(f)=K \cap A \subseteq \operatorname{Rad}(K)$ and $\operatorname{Ker}(g)=\frac{V}{A} \cap \frac{B}{A} \subseteq \operatorname{Rad}\left(\frac{V}{A}\right)$. Then 
$g \circ f: K \longrightarrow \frac{N}{B}$ is an epimorphism such that $K \cap B=\operatorname{Ker}(g \circ f) \subseteq \operatorname{Rad}(K)$ by [12, Lemma 1.1]. Finally, $N=K+B$.

Proposition 5. Let $M$ be a module and $K$ be a radical submodule of $M$. If $\frac{M}{K}$ has the property (CRE), then $M$ has the property (CRE).

Proof. Let $U$ be any cofinite extension of $M$. Then $\frac{U}{M}$ is finitely generated. So $\frac{\frac{U}{K}}{\frac{M}{K}} \cong \frac{U}{M}$ is finitely generated. Thus $\frac{U}{K}$ is a cofinite extension of $\frac{M}{K}$. By the hypothesis, there exists a submodule $\frac{V}{K}$ of $\frac{U}{K}$ such that $\frac{U}{K}=\frac{M}{K}+\frac{V}{K}$ and $\frac{M}{K} \cap \frac{V}{K} \subseteq \operatorname{Rad}\left(\frac{V}{K}\right)$. Then we have $U=M+V$. Since $K$ is radical, $M \cap V \subseteq \operatorname{Rad}(V)$. So $V$ is a $R a d$ supplement of $M$ in $U$. Therefore $M$ has the property (CRE).

Now we prove that the class of modules with the property (CRE) is closed under extensions if we impose certain conditions.

Theorem 2. Let

$$
0 \longrightarrow K \stackrel{f}{\longrightarrow} M \stackrel{g}{\longrightarrow} L \longrightarrow 0
$$

be a short exact sequence. Suppose that $K$ is radical. If $K$ and $L$ have the property (CRE), so does $M$. If the sequence splits, the converse holds.

Proof. Without loss of generality; we assume that $K \subseteq M$. Since $\frac{M}{K} \cong L$ has the property (CRE) and $K$ is radical, then we have $M$ has the property (CRE) by Proposition 5. On the other hand, suppose that the sequence splits. Then $M \cong K \oplus L$. Let $M$ be module with the property (CRE). So, $K$ and $L$ have the property (CRE) by Proposition 2.

Corollary 3. Let $M_{i}(i \in I)$ be any finite collection of radical modules and $M=$ $M_{1} \oplus M_{2} \oplus \ldots \oplus M_{n}$. Then $M$ has the property (CRE) iff $M_{i}$ has the property (CRE) for each $i=1,2, \ldots, n$.

Proof. The necessity follows from Proposition 2. Conversely, to prove that $M$ has the property (CRE) it is sufficient by induction on $n$ to prove that this is the case when $n=2$. Thus suppose $M=M_{1} \oplus M_{2}$. By using the following short exact sequence

$$
0 \longrightarrow M_{1} \longrightarrow M \longrightarrow M_{2} \longrightarrow 0 \text {. }
$$

Since $M_{1}$ is radical and $M_{1}, M_{2}$ have properties (CRE), then $M$ has the property (CRE) by Theorem 2.

Let $M$ be a module. A module $N$ is called maximal essential extension of $M$ if $N$ is an essential extension of $M$, and whenever $K$ is a proper extension of $N$, then $K$ is not an essential extension of $M([8])$.

Let $M$ be a module. Then there exists a module $N$ satisfying the following equivalent conditions: 
(1) $N$ is an essential injective extension of $M$.

(2) $N$ is a maximal essential extension of $M$.

A module $N$ satisfying above equivalent conditions is called injective envelope of $M$, denoted by $E(M)$ ([8, Theorem 2.21]).

Proposition 6. Let $M$ be a module.Then the following statements are equivalent.

(1) $M$ has a Rad-supplement in every cofinite essential injective extension.

(2) M has a Rad-supplement in every cofinite maximal essential extension.

Proof. (1) $\Longrightarrow(2)$ is clear.

(2) $\Longrightarrow$ (1) Let $N$ be any cofinite essential extension of $M, T$ be any cofinite maximal essential extension of $M, f: M \longrightarrow N, g: M \longrightarrow T$ be inclusion mappings and $I_{T}: T \longrightarrow T$ be an identity mapping. Then we have the following commutative diagram with $h$ necessarily monic:

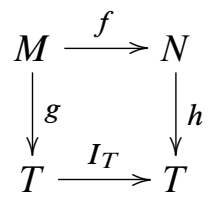

By the hypothesis, $M$ has a $R a d$-supplement in $T$, say $K$, that is, $M+K=T$ and $M \cap K \subseteq \operatorname{Rad}(K)$. Since $M \subseteq h(N)$, we obtain that $h(N)=h(N) \cap T=h(N) \cap$ $(M+K)=M+(h(N) \cap K)$. Now, taking any $n \in N$, we have $h(N)=m+h\left(n_{1}\right)=$ $h\left(m+n_{1}\right)$ where $m \in M$ and $h\left(n_{1}\right) \in h(N) \cap K$. So, $n=m+n_{1} \in M+h^{-1}(K)$ since $h$ is monic, and thus $M+h^{-1}(K)=N$. Moreover, $M \cap h^{-1}(K)=h^{-1}(M \cap$ $K) \subseteq \operatorname{Rad}\left(h^{-1}(K)\right)$. Since $h^{-1}(M)=M$ as $h$ is monic. Hence $h^{-1}(K)$ is a $\operatorname{Rad}$ supplement of $M$ in $N$.

Proposition 7. Let $M$ be a module with the property (CRE). If $M$ is noetherian, then $M$ has the property (CE).

Proof. Let $N$ be any cofinite extension of $M$. Since $M$ has the property (CRE), then there exists a submodule $K$ of $N$ such that $N=M+K$ and $M \cap K \subseteq \operatorname{Rad}(K)$. Since $M$ is noetherian, then $M \cap K$ is finitely generated. So it is easy to see that $M \cap K \ll K$. So $M$ has the property (CE).

A ring $R$ is called semiperfect if $\frac{R}{\operatorname{Rad}(R)}$ is semisimple and idempotents can be lifted modulo $\operatorname{Rad}(R)$. It is well known that a ring $R$ is semiperfect if and only if every left (or right) ideal has a supplement in ${ }_{R} R$ (or $R R$ ). A ring $R$ is called $R a d$ supplemented if every left (or right) ideal has a $R a d$-supplement in ${ }_{R} R$ (or ${ }_{R} R$ ) (see [2]). Clearly, every semiperfect ring is $R a d$-supplemented, but a $R a d$-supplemented ring need not be semiperfect in general (see [2]).

It is proved in [3, Proposition 2.11] that a ring $R$ is semiperfect if and only if every left $R$-module has the property (CE). Now, we give the characterization of the rings over which every module has the property (CRE). 
Theorem 3. The following statements are equivalent for a ring $R$ :

(1) $R$ is Rad-supplemented.

(2) ${ }_{R} R$ has ample Rad-supplements in every cofinite extension.

(3) $R R$ has ample Rad-supplements in every finitely generated extension.

(4) Every left $R$-module has the property (CRE).

(5) Every left R-module has the property (CREE).

Proof. (1) $\Longrightarrow$ (4) Let $M$ be an $R$-module and $N$ be a cofinite extension of $M$. Then there exists a finitely generated submodule $K$ of $N$ such that $N=M+K$. Since $R$ is a $R a d$-supplemented ring, then $M \cap K$ has a $R a d$-supplement in $K$, say $T$, i.e. $K=(M \cap K)+T$ and $(M \cap K) \cap T \subseteq \operatorname{Rad}(T)$ by [10, Theorem 3.7]. It follows that $N=M+T$ and $M \cap T \subseteq \operatorname{Rad}(T)$. This completes the proof.

(4) $\Longrightarrow$ (1) Suppose that every left $R$-module has the property (CRE). Then every left ideal of $R$ has a $R a d$-supplement in $R$ as a left $R$-module. Hence ${ }_{R} R$ is $R a d$ supplemented.

(2) $\Longrightarrow$ (1) If ${ }_{R} R$ has the property (CREE), it is $R a d$-supplemented by Corollary 2.
(5) $\Longrightarrow$
(4) and (5) $\Longrightarrow$
$(3) \Longrightarrow(2)$
(2) are clear.

A module $M$ over a ring $R$ is called uniserial if the lattice of submodules of $M$ is a chain and $M$ is called serial if $M$ is a direct sum of uniserial modules. A ring $R$ is called left (right) uniserial if the module ${ }_{R} R\left(R_{R}\right)$ is uniserial. $R$ is called a uniserial ring if $R$ is both right and left uniserial. A uniserial domain $R$ is called nearly simple if $\operatorname{Rad}(\mathrm{R})$ is the unique nonzero two sided ideal of $R$ and $\operatorname{Rad}^{2}(R) \neq 0$ [7].

The following example shows that a module with the property (CRE) need not be (CE).

Example 1. (See[2]) Let $G=\{f: \mathbb{Q} \longrightarrow \mathbb{Q} \mid f(t)=a t+b$ for $a, b \in \mathbb{Q}$ and $a>0\}$ be the group of affine linear functions on the field of rational numbers $\mathbb{Q}$. Choose any irrational number $\epsilon \in \mathbb{R}$ and set $P=\{f \in G \mid \epsilon \leq f(\epsilon)\}$ and $P^{+}=\{f \in G \mid \epsilon<f(\epsilon)\}$. Note that $P$, resp. $P^{+}$, defines a left order on $G$. Take an arbitrary field $F$ and consider the semigroup group ring $F[P]$ in which the right ideal $M=\sum_{g \in P} g F[P]$ is maximal. The set $F[P] \backslash M$ is a left and right ore set and the corresponding localization $R$ is a nearly simple uniserial domain. Taking any nonzero element $r \in R$, $S=\operatorname{End}\left(\frac{R}{r R}\right)$ is a $R a d$-supplemented ring which is not semiperfect. Since $S$ is a Rad-supplemented ring, then ${ }_{S} S$ has the property (CRE) by Theorem 3. It is clear that $S S$ has not the property (CE) by [3, Proposition 2.11]

The ring $R$ is called left hereditary if and only if all left ideals of $R$ are projective. It is well known that for a left hereditary ring $R$, every submodule of a free $R$-module is isomorphic to a direct sum of left ideals of $R$ and hence is projective.

Corollary 4. For a left hereditary ring $R$, the following statements are equivalent.

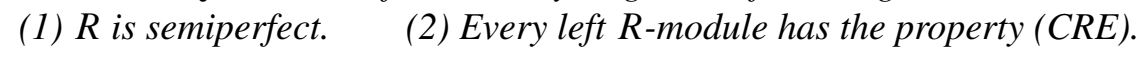


Proof. (1) $\Longrightarrow(2)$ Let $M$ be any $R$-module and $N$ be a cofinite extension of $M$. Then there exists a submodule $K$ of $N$ such that $N=M+K$. Since $K$ is finitely generated, then so is $\frac{N}{M} \cong \frac{K}{M \cap K}$. Therefore $\frac{N}{M}$ has a projective cover because $R$ is semiperfect. By [6, Lemma 4.40], there exists a submodule $L$ of $N$ such that $N=M+L$ and $M \cap L \ll L$. So $M \cap L \subseteq \operatorname{Rad}(L)$. Thus $M$ has the property (CRE).

(2) $\Longrightarrow(1)$ By Theorem 3, R is is Rad-supplemented. Applying [9, Corollary 2.22], it is semiperfect.

Acknowledgement. We would like to thank the referee for the valuable suggestions and comments.

\section{REFERENCES}

[1] R. Alizade, G. Bilhan, and P. F. Smith, "Modules whose maximal submodules have supplements," Commun. Algebra, vol. 29, no. 6, pp. 2389-2405, 2001.

[2] E. Büyükaşik and C. Lomp, "On a recent generalization of semiperfect rings," Bull. Aust. Math. Soc., vol. 78, no. 2, pp. 317-325, 2008.

[3] H. Çalışıcı and E. Türkmen, "Modules that have a supplement in every cofinite extension," Georgian Math. J., vol. 19, no. 2, pp. 209-216, 2012.

[4] J. Clark, C. Lomp, N. Vanaja, and R. Wisbauer, Lifting modules. Supplements and projectivity in module theory, ser. Frontiers in Mathematics. Basel: Birkhäuser, 2006.

[5] T. Y. Lam, Lectures on modules and rings, ser. Graduate Texts in Mathematics. New York: Springer, 1999, vol. 189.

[6] S. H. Mohamed and B. J. Müller, Continuous and discrete modules, ser. London Mathematical Society Lecture Note Series. Cambridge: Cambridge University Press, 1990, vol. 147.

[7] G. Puninski, "Some model theory over a nearly simple uniserial domain and decompositions of serial modules," J. Pure Appl. Algebra, vol. 163, no. 3, pp. 319-337, 2001.

[8] D. W. Sharpe and P. Vamos, Injective modules, ser. Cambridge Tracts in Mathematics and mathematical Physics. Cambridge: At the University Press, 1972, vol. 62.

[9] E. Türkmen and A. Pancar, "Some properties of Rad-supplemented modules," International Journal of the Physical Sciences, vol. 6, no. 35, pp. 7904-7909, 2011.

[10] E. Türkmen and A. Pancar, "On cofinitely Rad-supplemented modules," Int. J. Pure Appl. Math., vol. 53, no. 2, pp. 153-162, 2009.

[11] R. Wisbauer, Foundations of module and ring theory. A handbook for study and research. Revised and updated Engl. ed., ser. Algebra, Logic and Applications. Philadelphia: Gordon and Breach Science Publishers, 1991, vol. 3.

[12] W. Xue, "Characterizations of semiperfect and perfect rings," Publ. Mat., Barc., vol. 40, no. 1, pp. $115-125,1996$.

[13] H. Zöschinger, "Moduln, die in jeder Erweiterung ein Komplement haben," Math. Scand., vol. 35, pp. 267-287, 1975 .

Author's address

\section{Burcu Nişancı Türkmen}

Amasya University, Faculty of Art and Science, Department of Mathematics, 05100, Amasya, Turkey

E-mail address: burcunisancie@hotmail.com 